\title{
$100 \%$ Sustained Virological Response and Fibrosis Improvement in Real-Life Use of Direct Acting Antivirals in Genotype-1b Recurrent Hepatitis C following Liver Transplantation
}

\author{
Speranta Iacob $^{1,2}$, Razvan Cerban ${ }^{1,2}$, Corina Pietrareanu' ${ }^{1}$, Carmen Ester ${ }^{1,2}$, Razvan Iacob ${ }^{1,2}$, Cristian Gheorghe ${ }^{1,2}$, Irinel \\ Popescu $^{3}$, Liana Gheorghe $e^{1,2}$
}

1) Center for Digestive

Diseases and Liver

Transplantation, Fundeni

Clinical Institute;

2) Carol Davila University of

Medicine and Pharmacy;

3) Dan Setlacec Centre of

General Surgery and Liver

Transplantation, Fundeni

Clinical Institute,

Bucharest, Romania

\author{
Address for correspondence: \\ Liana Gheorghe, $\mathrm{MD}, \mathrm{PhD}$ \\ Center for Digestive Diseases \\ and Liver Transplantation, \\ Fundeni Clinical Institute, \\ Bucharest, Romania \\ Fundeni str 258, sector 2, \\ 022328, Bucharest, Romania \\ drlgheorghe@gmail.com
}

Received: 24.01 .2018 Accepted: 28.04.2018

\begin{abstract}
Background: Nowadays, interferon-free therapy using new direct-acting antivirals (DAA) has dramatically increased the cure rate across different $\mathrm{HCV}$-infected patient populations, including groups traditionally viewed as difficult-to-treat (patients with co-infections, cirrhosis and liver transplant - LT recipients) with marked improvement in safety and tolerability.

Aim: To present our experience with DAA therapy in LT recipients, as well as to compare pre- and posttreatment liver stiffness (LS) and noninvasive fibrosis scores.

Methods: Our cohort consisted of 89 patients with genotype 1 (GT1) recurrent hepatitis C after LT. Seventy six patients received ombitasvir/paritaprevir/ritonavir+dasabuvir+ribavirin and 13 sofosbuvir/ ledipasvir \pm ribavirin. Fibroscan ${ }^{\circ}$, FIB4 and APRI scores were performed in all patients before and 12 weeks after DAA therapy.

Results: We analyzed 45 (50.5\%) males and 44 (49.5\%) females with a mean age of $55 \pm 7.7$ years. Median time since LT was 20.9 months. At baseline, 53 (59.6\%) of patients had severe necroinflammation at Fibromax ${ }^{\circ}$; advanced fibrosis (F3, F4) was encountered in 35 (39.4\%) and grade 3 steatosis in 33 (37.1\%) of LT recipients. End of therapy (EOT) virological response (VR) was 100\%. Sustained virological response 12 weeks after therapy (SVR12) was $97.7 \%$ in the intention-to-treat analysis and $100 \%$ in per protocol analysis. There was a significant improvement in LS between antiviral therapy initiation and SVR12: $11.9 \pm 1.05 \mathrm{kPa}$ vs $8.8 \pm 0.6 \mathrm{kPa}$ $(\mathrm{p}<0.0001)$, as well as in APRI ( $2.7 \pm 0.3$ vs $0.4 \pm 0.05, \mathrm{p}<0.0001)$ and FIB4 ( $4.6 \pm 0.5$ vs $2.5 \pm 0.2, \mathrm{p}<0.0001)$ scores. Conclusions: In HCV positive recipients, DAA regimens are highly effective and safe. A significant decrease of LS by transient elastography and fibrosis non-invasive scores can be observed after successful therapy.
\end{abstract}

Key words: hepatitis C - liver transplantation - antiviral therapy.

Abbreviations: AE: adverse events; AFP: alpha fetoprotein; ALP: alkaline phosphatase; ALT: alanine aminotransferases; AST: aspartate aminotransferases; DAA: direct-acting antivirals; GGT: gamma glutamyl transpeptidase; GT1: genotype 1; HC: Hepatitis C; HCC: hepatocellular carcinoma; HCV: hepatitis C virus; HIV: human immunodeficiency virus; INR: international normalized ratio; ITT: intention to treat; LDV/SOF: ledipasvir/sofosbuvir; LLD: lower limits of detection; LLQ: lower limits of quantification; LS: liver stiffness; LSM: liver stiffness measurement; LT: liver transplant; NHIA: National Health Insurance Agency; OBV/ $\mathrm{PTV} / \mathrm{r}+\mathrm{DSV}$ : Ombitasvir/Paritaprevir/ritonavir plus dasabuvir (3D); RBV: ribavirin; SAE: serious adverse events; SOF+DAC \pm RBV: sofosbuvir+daclatasvir+ribavirin; SVR: sustained virological response; TE: transient elastography; VR: virological response.

\section{INTRODUCTION}

Hepatitis C (HC) is a common cause of liver cirrhosis, liver failure and hepatocellular carcinoma (HCC), and a frequent indication for liver transplantation (LT). Recurrent $\mathrm{HC}$ after LT is universal in those viremic at the time of transplantation and represents an important cause of graft failure, a need for retransplantation and low patient survival [1]. In the interferon era, patients with recurrent $\mathrm{HC}$ were considered difficult to treat due to the low rate (30-40\%) of sustained virological response (SVR), the poor tolerability and the high rate of therapy discontinuation due to side effects, including immune-mediated graft dysfunction [2]. New direct-acting antivirals (DAAs) have dramatically improved SVR rates in patients treated for recurrent HC. 
Nowadays, reported SVR rates in post-transplant setting ranged between 88 and 100\% [3-6], with good safety profiles. A high rate of virologic response (VR) (79\%) was also reported in patients with fibrosing cholestatic hepatitis after LT who were treated with interferon-free therapies [7]. DAAbased treatments for severe post-transplant $\mathrm{HC}$ recurrence significantly improve liver function, even if viral clearance has not been achieved and permit an excellent long-term survival. Several obstacles still need to be overcome including drug-drug interactions, DAA-resistant $\mathrm{HCV}$, treatment for decompensated cirrhosis and renal failure [8].

It has been shown that Ombitasvir/Paritaprevir/ritonavir plus dasabuvir $(\mathrm{OBV} / \mathrm{PTV} / \mathrm{r}+\mathrm{DSV})(3 \mathrm{D})$ plus ribavirin in LT recipients with recurrent $\mathrm{HCV}$ is an outcome-improving and cost-effective regimen for this population [9]. Currently, the $3 \mathrm{D}$ regimen is one of the elective DAAs recommended by international guidelines $[10,11]$ and it was the single one covered by the National Insurance Health Agency for HCV therapy in Romania in 2015-2016. We recently published our experience with the $3 \mathrm{D}$ regimen in compensated liver cirrhosis [12]. Starting with 2017, ledipasvir/sofosbuvir (LDV/SOF) regimen has been reimbursed for the use in LT recipients in our country.

The aim of this study was to present the first Romanian cohort and evaluate the effectiveness and safety of DAA regimens $(\mathrm{OBV} / \mathrm{PTV} / \mathrm{r}+\mathrm{DSV}$ plus ribavirin or $\mathrm{LDV} / \mathrm{SOF}$ with or without ribavirin) in treatment-experienced or naive patients with GT1 recurrent HC after LT in routine clinical practice.

\section{METHODS}

\section{Patients' selection}

Patients were included in this observational study according to the criteria for access to antiviral therapy established by the National Health Insurance Agency (NHIA): adult ( $>18$ years old), treatment experienced or naïve patients with recurrent $\mathrm{HC}$ after LT irrespective of fibrosis stage assessed by Fibromax ${ }^{\circledR}$ (Biopredictive), and positive HCV RNA. Consecutive patients transplanted in our center were included in this non-interventional, prospective cohort. All patients had at least a liver biopsy performed after LT confirming the diagnosis of recurrent HC. All patients enrolled in the study had liver stiffness measurement (LSM) by transient elastography (TE) with Fibroscan ${ }^{\circledR}$ performed at baseline, end of treatment (EOT) and 12 weeks after therapy. Plasma HCV RNA levels were measured using the Roche TaqMan real-time reverse transcriptase-PCR assay version 2.0 with a lower limits of quantification (LLQ) and detection (LLD) of $15 \mathrm{IU} / \mathrm{mL}$. All patients underwent genotyping for hepatitis $\mathrm{C}$ virus $(\mathrm{HCV})$ and all proved to be GT 1b. Patients' enrolment started in December 2015 and ended in June 2017 in order to allow for an adequate follow-up time.

Exclusion criteria were: less than 3 months after LT without stable immunosuppression medication, evidence of recurrent HCC after LT, concomitant medication that is contraindicated according to manufacturer's recommendations, patients with human immunodeficiency virus (HIV) coinfection, current pregnancy and lactation, unable/unwilling to use contraception during the treatment and 6 months after discontinuation, platelet count $<25,000 / \mathrm{mm}^{3}$. Patients were enrolled for treatment with $\mathrm{OBV} / \mathrm{PTV} / \mathrm{r}+\mathrm{DSV}$ plus ribavirin $(\mathrm{RBV})$ or LDV/SOF plus RBV according to the therapeutic guidelines of the Protocol Expert Committee of Ministry of Health and NHIA. Written informed consent was obtained from each patient for initiation, monitoring and follow-up during therapy. The study was conducted in accordance with the principles of the Declaration of Helsinki.

\section{Exposure to therapy and follow-up}

Seventy-six patients received therapy with $\mathrm{OBV} / \mathrm{PTV} / \mathrm{r}$ (Viekirax 12.5mg/75mg/50mg; Abbvie Deutschland Gmbh \& Co Ludwigshafen, Germany) $25 \mathrm{mg} / 150 \mathrm{mg} / 100 \mathrm{mg} /$ day in a single daily dose and DSV (Exviera $250 \mathrm{mg}$; Abbvie Deutschland Gmbh \& Co Ludwigshafen, Germany) $500 \mathrm{mg} /$ day divided in two daily doses for 24 weeks during the years 2015-2016. In 2017, 13 patients received therapy with LDV/ SOF (Harvoni 90mg/400mg; Gilead Sciences, Foster City, CA) with or without RBV for 12 or 24 weeks, respectively. All patients received RBV according to body weight $(<75 \mathrm{~kg}, 1000$ $\mathrm{mg} /$ day; $>75 \mathrm{~kg}, 1200 \mathrm{mg} /$ day). In case of significant laboratory abnormalities at baseline (anemia, thrombocytopenia or chronic renal failure) treatment was started with a lower dose of RBV, according to the product characteristics. RBV dose was modified or discontinued during therapy in patients who developed severe adverse events or laboratory abnormalities. Patients were assessed clinically and biologically at baseline, week $4,8,12,16,20,24$ and 12 weeks after therapy. Baseline clinical data included gender, age, body weight/body mass index, HCV GT, necroinflammatory, fibrosis and steatosis status according to Fibromax ${ }^{\oplus}$, LSM by TE with Fibroscan ${ }^{\circledR}$, other noninvasive scores for fibrosis (APRI and FIB-4 scores), treatment history (previous interferon exposure), comorbidities, and concomitant medication. Transient elastography and other noninvasve scores were performed also at SVR12. Liver cirrhosis was established based on noninvasive tests (TE and/or Fibromax and/or APRI/FIB-4) or a previous liver biopsy showing F4 METAVIR and ultrasound liver aspect as well as biochemical results.

Laboratory data included HCV RNA level (performed at baseline, week 4, EOT and SVR), liver function tests (bilirubin, alanine - ALT and aspartate aminotransferases - AST, alkaline phosphatase - ALP, gamma glutamyl transpeptidase - GGT, international normalized ratio - INR, albumin), platelets and leucocytes count, haemoglobin concentration, serum creatinine and creatinine clearance, alpha fetoprotein (AFP) at baseline, and at every established visit. Immunosuppression was adjusted initially according to the current recommendations [13]: tacrolimus $0.5 \mathrm{mg}$ every 7 days and cyclosporine at onefifth the total daily dose administered once daily and then adjusted according to the desired trough concentrations (C trough) during 3D treatment. For patients receiving LDV/SOF, trough levels of immunosuppressive drugs were monitored and the dose adjusted accordingly. Other concomitant immunosuppressive medications such as mycophenolate mofetil were stopped during antiviral therapy. 


\section{Efficacy and safety analysis}

Efficacy primary endpoint was assessed by the percentage of patients achieving SVR12, defined as an undetectable HCV RNA 12 weeks after the last dose of antiviral medication. Secondary assessments included the percentage of patients with ontreatment virologic failure and post-treatment relapse. Samples were analyzed according to an intention to treat (ITT) but also per protocol analysis. Treatment-emergent adverse events (AE), serious adverse events (SAE), as well as premature treatment discontinuations during the treatment period and 12 weeks after EOT were recorded carefully and considered for safety analysis.

\section{Statistical analysis}

Continuous variables were reported as a mean \pm standard deviation, while categorical variables were reported as frequencies and percentages. Comparisons were made by chi-square-test or McNemar test for qualitative variables; Mann-Whitney-U and Wilcoxon tests were used to compare continuous data. Friedman or Kruskal-Wallis test were performed for global comparison of quantitative variables. The degree of association between the variables was calculated using Rank correlation (Spearman correlation coefficient). All statistical tests were two-sided and a level of $\mathrm{P}<0.05$ was used to indicate statistical significance. All statistical analyses were carried out using NCSS 11.0 (LLC, Kaysville, Utah, USA).

\section{RESULTS}

\section{Patients' characteristics}

The demographical and clinical baseline characteristics are shown in Table I. A total of 89 naïve and treatment experienced patients with GT $1 \mathrm{~b}$ recurrent $\mathrm{HC}$ were followed up prospectively. Seventy six patients received OBV/PTV/r+DSV+RBV for 24 weeks, $12 \mathrm{LDV} / \mathrm{SOF}+\mathrm{RBV}$ for 12 weeks and one Ribavirinintolerant patient received LDV/SOF for 24 weeks. Eighteen patients $(20.2 \%)$ had HCC prior to LT.

Table I. Baseline demographical and clinical characteristics of the transplanted patients ( $\mathrm{N}=89$ patients)

\begin{tabular}{ll}
\hline Characteristic & Value (mean or n/\%) \\
\hline Mean age (years) & $55 \pm 7.7$ \\
Gender (males) & $45(50.5 \%)$ \\
Mean BMI (kg/m $\left.{ }^{2}\right)$ & $26.3 \pm 4.4($ range $17.3-35.7)$ \\
Mean viral load (UI/mL) & $2,779,089 \pm 436,200$ \\
Fibromax & \\
$\quad$ A3/A2/A1/A0 (\%) & $59.6 / 21.3 / 14.6 / 4.5$ \\
$\quad$ F4/F3/F2/F1/F0 (\%) & $31.5 / 7.9 / 36 / 15.7 / 9$ \\
$\quad$ Mean liver stiffness (kPa) & $37.1 / 30.3 / 20.2 / 12.4$ \\
Diabetes mellitus & $12.5 \pm 1.2$ \\
$\quad$ Insulin-dependent & $30(33.7 \%)$ \\
Cryoglobulinemia & $17(19.1 \%)$ \\
Tacrolimus/Cyclosporin/Sirolimus & $12(13.4 \%)$ \\
Mean value of creatinine eGFR & $73(82 \%) / 13(14.7 \%) / 3(3.3 \%)$ \\
(ml/min) & $86.2 \pm 35.6$ \\
IFN-based treatment experience & $30(33.7 \%)$ \\
patients post-LT & \\
Time from LT (months) & $20.9(\mathrm{range} 3.4-176.9)$ \\
\hline
\end{tabular}

Significant difference was found in LSM between the three grades of inflammation $(6.2 \mathrm{kPa}$ for $\mathrm{A} 0-\mathrm{A} 1,6.6 \mathrm{kPa}$ for $\mathrm{A} 2$ and $10.7 \mathrm{kPa}$ for $\mathrm{A} 3, \mathrm{p}=0.00002)$, but not between patients with different grades of steatosis at Fibromax ${ }^{\circledR}(6.6 \mathrm{kPa}$ for $\mathrm{S} 0-\mathrm{S} 1,8.5 \mathrm{kPa}$ for S2 and $10.1 \mathrm{kPa}$ for $\mathrm{S} 3, \mathrm{p}=0.15$ ). There was a significant correlation between baseline LSM and Fibrotest of Fibromax $^{\circledast}(\mathrm{r}=0.47, \mathrm{p}=0.0001)$, APRI $(\mathrm{r}=0.41, \mathrm{p}=0.0001)$ and FIB4 ( $\mathrm{r}=0.29, \mathrm{p}=0.01)$.

Asymptomatic mixed cryoglobulinemia was present in 12 (13.4\%) patients; however, clinical manifestations (renal impairment and neuropathy) were present in 2 patients. All patients with liver cirrhosis (26 patients, 29.2\%) belonged to Child Pugh A, except 1 patient with HCV recurrent liver cirrhosis who belonged to Child Pugh B class.

\section{Efficacy analysis}

HCV RNA was undetectable in 55 (61.8\%) of patients at week 4 . The overall EOT rate was $98.8 \%$ (88/89 patients) and, respectively, the overall SVR12 rate was $97.7 \%(87 / 89)$ in an ITT analysis. Per protocol, EOT virological response was $100 \%$ and SVR 12 rate was $100 \%$.

Table II summarizes the differences between baseline and different time points during antiviral therapy (week 4, 12, 24 and posttreatment week 12).

Table II. Median laboratory parameters changes between baseline and 12 weeks post-treatment

\begin{tabular}{lccccc}
\hline Variable & Baseline & Week 4 & $\begin{array}{c}\text { EOT } \\
\text { (Week 12/24) }\end{array}$ & SVR12 & $\begin{array}{c}\text { P } \\
\text { value }\end{array}$ \\
\hline $\begin{array}{l}\text { Hemoglobin } \\
\text { (g/dL) }\end{array}$ & 13.9 & 12.4 & 12.3 & 14.9 & 0.24 \\
$\begin{array}{l}\text { ALT (IU/L) } \\
\text { AST (IU/L) }\end{array}$ & 186 & 34.1 & 30.5 & 23.7 & 0.008 \\
GGT (IU/L) & 120.7 & 25.5 & 25.4 & 23.3 & 0.0005 \\
ALP (IU/L) & 173.5 & 127.3 & 130 & 120.7 & 0.03 \\
$\begin{array}{l}\text { Total bilirubin } \\
\text { (mg/dL) }\end{array}$ & 1.11 & 1.40 & 0.86 & 0.84 & 0.007 \\
Albumin (g/dL) & 4.05 & 4.36 & 4.50 & 4.49 & 0.0002 \\
$\begin{array}{l}\text { Creatinine } \\
\text { (mg/dL) }\end{array}$ & 1.03 & 1.06 & 1.18 & 1.06 & 0.002 \\
INR & 1.10 & 1.07 & 1.13 & 1.15 & 0.13 \\
\hline
\end{tabular}

At EOT, 5 (41.66\%) out of 12 patients positive for cryoglobulins became negative after virological response. Alpha fetoprotein value decreased significantly between baseline and SVR $(19.9 \pm 7.3 \mathrm{ng} / \mathrm{mL}$ vs $3.2 \pm 0.2 \mathrm{ng} / \mathrm{mL}, \mathrm{p}=0.02)$. Sustained biochemical response (ALT $<\mathrm{UNV}$ ) was encountered in $83.1 \%(74 / 89)$ of patients.

Liver stiffness measurement and noninvasive scores

Liver stiffness measurement significantly decreased at EOT and SVR12 compared to baseline (Fig. 1).

Table III (A and B) summarizes the evolution of LSM and other noninvasive scores from baseline to SVR12. Initial values of LSM $(11.9 \pm 1.05$ vs $8.8 \pm 0.6 \mathrm{kPa}, \mathrm{p}<0.0001)$, FIB-4 $(4.6 \pm 0.5$ vs $2.5 \pm 0.2, \mathrm{p}<0.0001)$ and APRI scores $(2.7 \pm 0.3$ vs $0.4 \pm 0.05$, $\mathrm{p}<0.0001)$ decreased significantly at SVR12. The decrease of 
Table III A and B. Evolution of liver stiffness measured (LSM) by TE, APRI and FIB-4 scores between baseline and SVR according to inflammation grade (A) and steatosis (B) at baseline

\begin{tabular}{lllllll}
\hline Variable & Baseline (A3) & SVR12 (A3) & P value & Baseline (A0-A2) & SVR12 (A0-A2) & P value \\
\hline LSM & $13.3 \pm 1.2$ & $9.6 \pm 0.7$ & $<0.0001$ & $9.8 \pm 1.9$ & $7.6 \pm 1.2$ & 0.01 \\
APRI & $4.1 \pm 0.5$ & $0.5 \pm 0.07$ & $<0.0001$ & $0.8 \pm 0.1$ & $0.4 \pm 0.04$ & $<0.0001$ \\
FIB-4 & $6 \pm 0.7$ & $2.7 \pm 0.2$ & $<0.0001$ & $2.8 \pm 0.4$ & $2.2 \pm 0.2$ & 0.006 \\
\hline & & & & & \\
\hline Variable & Baseline (S3) & SVR12 (S3) & P value & Baseline (S0-S2) & SVR12 (S0-S2) & P value \\
\hline LSM & $12.4 \pm 1.5$ & $9.2 \pm 1.07$ & 0.0001 & $11.5 \pm 1.4$ & $8.5 \pm 0.8$ & 0.0002 \\
APRI & $2.3 \pm 0.5$ & $0.5 \pm 0.1$ & $<0.0001$ & $3.05 \pm 0.5$ & $0.5 \pm 0.04$ & $<0.0001$ \\
FIB-4 & $4.3 \pm 0.7$ & $2.01 \pm 0.2$ & 0.0006 & $4.8 \pm 1.0$ & $2.5 \pm 0.3$ & 0.0064 \\
\hline
\end{tabular}

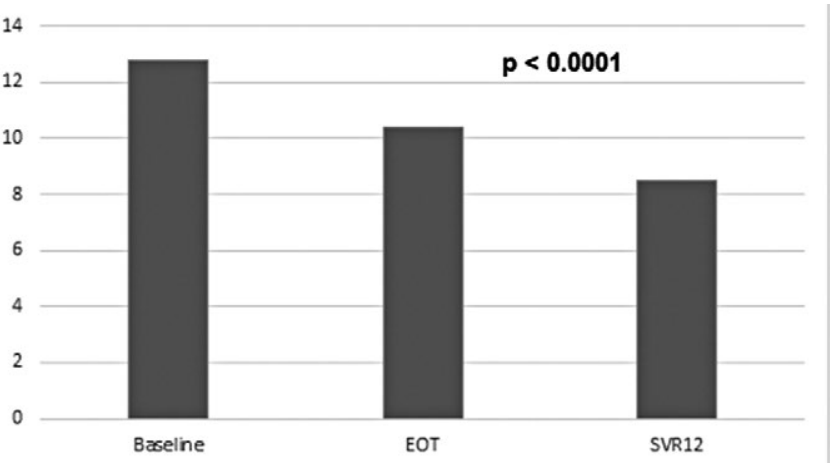

Fig. 1. Liver stiffness decrease between baseline, EOT and SVR

LSM, FIB-4 and APRI scores was more pronounced in patients with severe inflammation and steatosis at Fibromax ${ }^{\otimes}$ at the beginning of antiviral therapy.

\section{Safety}

Only one patient $(1.3 \%)$ stopped therapy before EOT in the OBV/PTV/r+DSV group. The following SAEs led to EOT in this patient: cholangitis with jaundice, renal dysfunction, Clostridium difficile infection after 6 weeks of therapy. However, this patient had undetectable HCV RNA after 4 weeks of antiviral therapy and remained HCV RNA negative 12 and 24 weeks after stopping 3D therapy. One patient reached EOT with undetectable HCV RNA, but died within one week after EOT. He received SOF/LDV and died due to sepsis after jejunal perforation through cytomegalovirus colitis. In the SOF/LDV group no other SAE was reported.

Adverse events were present in $64(61.8 \%)$ patients. Anemia was encountered in 41 (47.1\%) patients. No patient developed grade 3 anemia and none received blood transfusions; ribavirin was decreased in all patients. Thirty-nine (42.7\%) patients had significant hyperuricemia during antiviral therapy with the need to take allopurinol. In the $3 \mathrm{D}$ group of patients the following SAEs were reported: urinary infections (7 patients); aggravating renal dysfunction (2 patients); cholangitis (4 patients). One patient developed adenocarcinoma of the pancreas after EOT, but this event was unrelated to antiviral medication. He received chemotherapy and survived one more year after SVR.

Four patients had an increase of aminotransferase levels after EOT, but 3 patients showed good response to the increase of immunosuppression therapy and only one remained with persistent variable hepatocytolisis (liver biopsy showed features of steatohepatitis, without signs of rejection). Decompensation of liver cirrhosis did not occur in any patient. The single patient with Child Pugh B liver cirrhosis who received SOF/ LDV improved to Child Pugh class A, with normalization of total bilirubin and serum albumin until the end of therapy. No HCC was reported in any of our patients during or after antiviral therapy, in the follow-up period until December 2017.

\section{DISCUSSION}

This is a relatively large cohort of LT recipients with GT1 recurrent $\mathrm{HC}$ receiving DAA therapy in a single transplant center, providing real-world data on the efficacy and safety of DAA therapy, especially OBV/PTV/r+DSV that had been previously reported to be used only in F0-F2 fibrosis stage after LT [14]. In contrast to the clinical trials [6,14-16], this population included patients with significant fibrosis $(\geq \mathrm{F} 2$ METAVIR in $75.4 \%$ of patients). The SVR 12 rate of $100 \%$ is impressive, even higher than that obtained in clinical trials. In CORAL-I study [14], the combination of OBV/PTV/r+DSV+RBV for 24 weeks was investigated in $34 \mathrm{LT}$ recipients with GT1 infection and achieved 97\% SVR12. The ALLY-1 study [16], combining sofo sbuvir+daclatasvir+ribavirin $(\mathrm{SOF}+\mathrm{DAC} \pm \mathrm{RBV})$, showed $94 \%$ SVR12 (30\% patients with liver graft cirrhosis).

In a large real-life cohort of 412 patients (HCV-TARGET study) [17], sustained viral clearance was achieved in $96 \%$ of $\mathrm{SOF} / \mathrm{LDV} \pm \mathrm{RBV}$-treated patients, $92 \%$ of $\mathrm{OBV} / \mathrm{PTV} / \mathrm{r}+\mathrm{DSV}$ treated patients and $100 \%$ of $S O F+D A C \pm R B V$-treated patients. Safety and tolerability of these antivirals were excellent with an overall rate of treatment discontinuation of $1.6 \%$ due to an adverse event in Saxena's paper [17] and 1.3\% in our study. However, the most common adverse events occurred in $75.8 \%$ of patients in the HCV-TARGET study and in only $61.8 \%$ of our cohort.

The Italian Compassionate use of Sofosbuvir (ITACOPS) [18] showed an SVR rate of only $86.7 \%$ in patients receiving SOF/RBV for 24 weeks, but of $98.3 \%$ when simeprevir or DAC was added to SOF/RBV. In addition, in patients with fibrosing cholestatic hepatitis, SVR12 rates were $100 \%$. Another reallife study [19] reporting SVR12 of 100\%, similar to ours, was performed on 35 GT1 HCV LT recipients, who received OBV/ $\mathrm{PTV} / \mathrm{r}+\mathrm{DSV} \pm \mathrm{RBV}$, most (77.1\%) with fibrosis stage $\geq 2$. In the AMBER-CEE study [19], safety was excellent, adverse events being mostly mild and transient, as in our larger cohort. 
All these results strengthen the success and simplicity of the current $\mathrm{HCV}$ antiviral therapy in the transplant setting, even in advanced fibrosis. Cirrhosis or fibrosing cholestatic hepatitis are no more negative predictive factors of nonresponse in the era of the new generation direct antivirals.

Noninvasive tools have been proposed to stage liver fibrosis also after LT, including serum biomarkers based on readily available parameters, such as FIB-4, APRI score and measurement of LS by TE [20]. Some of them have also been specifically validated in LT recipients and the reported accuracy is similar to that in the pre-LT setting [21-24]. However, TE had a higher diagnostic accuracy than APRI and FIB-4 in order to detect significant liver fibrosis in the post-transplant setting [20]. Recently, repeated TE in real-life clinical practice appears to have a practical role in monitoring LT patients [25]. Because TE proved to be more accurate than biomarkers (FibroTest ${ }^{\circ}$, Benlloch score, Hepascore, APRI, FIB-4 etc) for the early detection of advanced fibrosis [20,24], we performed systematically TE in our center also in recurrent $\mathrm{HCV}$ infection according to an established protocol and then during antiviral therapy. Similarly to non-transplanted patients, TE and other noninvasive tests significantly decreased at SVR12, and the decrease was significantly greater in patients with a high baseline level of inflammation [12, 26-28]. However, in LT recipients this is the third article published so far documenting the decrease of LS assessed by TE after DAA. In the study by Martini et al. [29], among the 77 responders to sofosbuvirbased regimens (27 F3, 50 F4) who underwent elastography at baseline and at the end of follow-up (48 weeks after end of therapy), 39 (50.6\%; $18 \mathrm{~F} 3,21 \mathrm{~F} 4)$ exhibited a regression in fibrosis stage. The same was true for APRI and FIB- 4 indexes that showed significant improvement at 24 and 48 weeks after therapy. Similar to our results, another recently published article [30] that evaluated $23 \mathrm{LT}$ patients with recurrent HC treated for 24 weeks with $\mathrm{OBV} / \mathrm{PTV} / \mathrm{r}+\mathrm{DSV} \pm \mathrm{RBV}$ combination therapy, showed that shear-wave elastography and also APRI and FIB-4 were successfully used to monitor the beneficial therapeutic effects of DAAs in hepatitis $C$ recurrence following LT. Mauro et al. [31] proved that $67 \%$ of the cohort presented fibrosis regression; HVPG, liver stiffness and ELF score significantly decreased after SVR. There are also few previous studies [32-33] reporting histological and clinical benefits, associated with improvement of patient and graft survival after SVR in LT recipients. This finding was in accordance with our study showing no decompensation of liver cirrhosis even in the context of a protease inhibitor administration and improvement of liver function in one patient.

The higher decrease of LS and other noninvasive scores in LT recipients based on improvement of liver steatosis is similar to a very recent study performed in 57 Japanese HCV patients with SVR following DAA showing reduction of both liver fibrosis and steatosis [34].

A limitation of our study is that TE was validated and cutoffs were established in viremic LT recipients. However, it was demonstrated that graft survival significantly improved in patients with LSM $\geq 8.7 \mathrm{kPa}$ at 1 year after LT who achieved SVR [35]. In addition, no association was observed between outcomes and LSM at 12 months in non-HCV LT patients. New studies are required to prove that this conclusion is valid also for HCV recipients who clear the virus. Another weak point of this study is the lack of performing Fibromax ${ }^{\circ}$ also at SVR12 in order to document its decrease according to other noninvasive tests (FibroTest ${ }^{\circledR}$ and also Actitest ${ }^{\circ}$ ). In addition, the absence of a histological paired evaluation at baseline and SVR12 is another impediment in demonstrating fibrosis regression. Further studies with serial liver biopsies, following $\mathrm{HCV}$ cured recipients with advanced chronic liver disease for a longer period of time, should be performed.

\section{CONCLUSIONS}

Our national cohort of LT recipients proved once more that real-life use, even of more difficult to use new antivirals such as OBV/PTV/r+DSV, in patients with advanced fibrosis, has good virological and clinical outcomes.

Although fibrosis improvement documented by decreasing of LS values may be, at least in part, explained by disappearance of inflammation after viral control, this is not the only mechanism, as demonstrated by the decrease of TE and fibrosis scores also in LT recipients without significant activity scores at baseline. Fibrosis reversibility is possible after successful interferon-free therapy in the LT setting and it may continue to improve over time.

\section{Conflicts of interest: None to declare.}

Authors' contribution: L.G., S.I., I.P. initiated and designed the study; S.I. centralized the data; S.I., R.I. performed statistical analysis; S.I., L.G. wrote the paper; L.G., S.I., R.C., C.P., C.E., C.G., R.I. provided the patients that were analyzed and gathered patient data; C.G. and I.P. revised the paper.

\section{REFERENCES}

1. Coilly A, Roche B, Samuel D. Current management and perspectives for HCV recurrence after liver transplantation. Liver Int 2013;33(Suppl 1):56-62. doi:10.1111/liv.12062

2. Kuo A, Terrault NA. Management of hepatitis $C$ in liver transplant recipients. Am J Transplant 2006;6:449-458. doi:10.1111/j.16006143.2005.01202.x

3. Lutchman G, Nguyen NH, Chang CY, et al. Effectiveness and tolerability of simeprevir and sofosbuvir in nontransplant and post-liver transplant patients with hepatitis $\mathrm{C}$ genotype 1. Aliment Pharmacol Ther 2016;44:738-746. doi:10.1111/apt.13761

4. Khemichian S, Lee B, Kahn J, et al. Sofosbuvir and Simeprevir Therapy for Recurrent Hepatitis C Infection after Liver Transplantation. Transplant Direct 2015;1:e21. doi:10.1097/TXD.0000000000000531

5. Coilly A, Fougerou-Leurent C, de Ledinghen V, et al. Multicentre experience using daclatasvir and sofosbuvir to treat hepatitis $\mathrm{C}$ recurrence - The ANRS CUPILT study. J Hepatol 2016;65:711718. doi:10.1016/j.jhep.2016.05.039

6. Kwok RM, Ahn J, Schiano TD, et al. Sofosbuvir plus ledispasvir for recurrent hepatitis $\mathrm{C}$ in liver transplant recipients. Liver Transpl 2016;22:1536-1543. doi:10.1002/1t.24614

7. Vukotic R, Conti F, Fagiuoli S, et al. Long-term outcomes of direct acting antivirals in post-transplant advanced hepatitis $\mathrm{C}$ virus recurrence and fibrosing cholestatic hepatitis. J Viral Hepat 2017;24:858-864 doi:10.1111/jvh.12712 
8. Ueda Y, Uemoto S. Interferon-free therapy for hepatitis $C$ in liver transplant recipients. Transplantation 2016;100:54-60. doi:10.1097/ TP.0000000000000860

9. Saab S, Gonzalez YS, Huber C, Wang A, Juday T. Cost-effectiveness of Ombitasvir/Paritaprevir/Ritonavir, Dasabuvir + Ribavirin for US Post-Liver Transplant Recurrent Genotype 1 HCV. Liver Int 2016;36:515-521. doi:10.1111/liv.13033

10. European Association for the Study of the Liver. EASL Recommendations on Treatment of Hepatitis C 2016. J Hepatol 2017;66:153-194. doi:10.1016/j.jhep.2016.09.001

11. AASLD/IDSA HCV Guidance Panel. Hepatitis C guidance: AASLDIDSA recommendations for testing, managing, and treating adults infected with hepatitis C virus. Hepatology 2015;62:932-954. doi:10.1002/hep. 27950

12. Gheorghe L, Iacob S, Curescu M, et al. Real-Life Use of 3 Direct-Acting Antiviral Regimen in a Large Cohort of Patients with Genotype-1b HCV Compensated Cirrhosis. J Gastrointestin Liver Dis 2017;26:275281. doi:10.15403/jgld.2014.1121.263.iac

13. Badri PS, Parikh A, Coakley EP, et al. Pharmacokinetics of Tacrolimus and Cyclosporine in Liver Transplant Recipients Receiving 3 DirectActing Antivirals as Treatment for Hepatitis C Infection. Ther Drug Monit 2016;38:640-645. doi:10.1097/FTD.0000000000000315

14. Kwo PY, Mantry PS, Coaldey E, et al. An interferon-free antiviral regimen for HCV after liver transplantation. N Engl J Med 2014;371:2375-2382. doi:10.1056/NEJMoa1408921

15. Manns M, Forns X, Samuel D, et al. G02: Ledipasvir/sofosbuvir and ribavirin is safe and efficacious in decompensated and post liver transplantation patients with HCV infection: preliminary results of the prospective Solar 2 trial. J Hepatol 2015;62(Suppl 2):S187-S188. doi:10.1016/S0168-8278(15)30003-9

16. Poordad F, Schiff ER, Vierling JM, et al. L08:Daclatasvir, sofosbuvir and ribavirin combination for HCV patients with advanced cirrhosis or posttransplant recurrence: phase 3 ALLY-1 study. J Hepatol 2015;62(Suppl 2):S261-S262. doi:10.1016/S0168-8278(15)30154-9

17. Saxena V, Khungar V, Verna EC, et al. Safety and efficacy of current direct-acting antiviral regimens in kidney and liver transplant recipients with hepatitis C: Results from the HCV-TARGET Study. Hepatology 2017;66:1090-1101. doi:10.1002/hep.29258

18. Carrai P, Morelli C, Cordone G, et al. The Italian compassionate use of sofosbuvir observational cohort study for the treatment of recurrent hepatitis C: clinical and virological outcomes. Transpl Int 2017;30:12531265. doi:10.1111/tri.13018

19. Tronina O, Durlik M, Wawrzynowicz-Syczewska M, et al. Real-World Safety and Efficacy of Ombitasvir/Paritaprevir/ Ritonavir/+Dasabuvir \pm Ribavirin (OBV/PTV/r/+DSV \pm RBV) Therapy in Recurrent Hepatitis C Virus (HCV) Genotype 1 Infection Post-Liver Transplant: AMBER-CEE Study. Ann Transplant 2017;22:199-207. doi:10.12659/AOT.903535

20. Bhat M, Tazari M, Sebastiani G. Performance of transient elastography and serum fibrosis biomarkers for non-invasive evaluation of recurrent fibrosis after liver transplantation: A meta-analysis. PLoS One 2017;12:e0185192. doi:10.1371/journal.pone.0185192

21. Carrión JA, Fernández-Varo G, Bruguera M, et al. Serum fibrosis markers identify patients with mild and progressive hepatitis $\mathrm{C}$ recurrence after liver transplantation. Gastroenterology 2010;138:147158. doi:10.1053/j.gastro.2009.09.047
22. Cholongitas E, Tsochatzis E, Goulis J, Burroughs AK. Noninvasive tests for evaluation of fibrosis in HCV recurrence after liver transplantation: a systematic review. Transpl Int 2010;23:861-870. doi:10.1111/j.14322277.2010.01142.x

23. Adebajo CO, Talwalkar JA, Poterucha JJ, Kim WR, Charlton MR. Ultrasound-based transient elastography for the detection of hepatic fibrosis in patients with recurrent hepatitis $\mathrm{C}$ virus after liver transplantation: a systematic review and meta-analysis. Liver Transpl 2012;18:323-331. doi:10.1002/lt.22460

24. Beckebaum S, Iacob S, Klein CG, et al. Assessment of allograft fibrosis by transient elastography and noninvasive biomarker scoring systems in liver transplant patients. Transplantation 2010;89:983-993. doi:10.1097/ TP.0b013e3181cc66ca

25. Rinaldi L, Valente G, Piai G. Serial Liver Stiffness Measurements and Monitoring of Liver-Transplanted Patients in a Real-Life Clinical Practice. Hepat Mon 2016;16:e41162. doi:10.5812/hepatmon.41162

26. Singh S, Facciorusso A, Loomba R, Falck-Ytter YT. Magnitude and Kinetics of Decrease in Liver Stiffness After Antiviral Therapy in Patients With Chronic Hepatitis C: A Systematic Review and Meta-analysis. Clin Gastroenterol Hepatol 2018;16:27-38.e4. doi:10.1016/j.cgh.2017.04.038

27. Sporea I, Lupuşoru R, Mare R, et al. Dynamics of liver stiffness values by means of transient elastography in patients with HCV liver cirrhosis undergoing interferon free treatment. J Gastrointestin Liver Dis 2017;26:145-150. doi:10.15403/jgld.2014.1121.262.dyn

28. Knop V, Hoppe D, Welzel T, et al. Regression of fibrosis and portal hypertension in $\mathrm{HCV}$-associated cirrhosis and sustained virologic response after interferon-free antiviral therapy. J Viral Hepat 2016;23:994-1002. doi:10.1111/jvh.12578

29. Martini S, Sacco M, Strona S, et al. Impact of viral eradication with sofosbuvir-based therapy on the outcome of post-transplant hepatitis C with severe fibrosis. Liver Int 2017;37:62-70. doi:10.1111/liv.13193

30. Korda D, Lenard ZM, Gerlei Z, et al. Shear-wave elastography for the assessment of liver fibrosis in liver transplant recipients treated for hepatitis C virus recurrence. Eur J Gastroenterol Hepatol 2018;30:27-32. doi:10.1097/MEG.0000000000001003

31. Mauro E, Crespo G, Montironi C, et al. Portal pressure and liver stiffness measurements in the prediction of fibrosis regression after SVR in recurrent hepatitis C. Hepatology 2018;67:1683-1694. doi:10.1002/hep.29557

32. Berenguer M, Palau A, Aguilera V, Rayón JM, Juan FS, Prieto M. Clinical benefits of antiviral therapy in patients with recurrent hepatitis C following liver transplantation. Am J Transplant 2008;8:679-687. doi:10.1111/j.1600-6143.2007.02126.x

33. Bizollon T, Ahmed SN, Radenne S, et al. Long term histological improvement and clearance of intrahepatic hepatitis $C$ virus RNA following sustained response to interferon-ribavirin combination therapy in liver transplanted patients with hepatitis $C$ virus recurrence. Gut 2003;52:283-287.

34. Kobayashi N, Iijima H, Tada T, et al. Changes in liver stiffness and steatosis among patients with hepatitis $\mathrm{C}$ virus infection who received direct-acting antiviral therapy and achieved sustained virological response. Eur J Gastroenterol Hepatol 2018;30:546-551. doi:10.1097/ MEG.0000000000001106

35. Crespo G, Lens S, Gambato $M$, et al. Liver stiffness 1 year after transplantation predicts clinical outcomes in patients with recurrent hepatitis C. Am J Transplant 2014;14:375-383. doi:10.1111/ajt.12594 